\title{
EL MORFEMA -FU DEL MAPUDUNGUN: LA CODIFICACIÓN GRAMATICAL DEL ANTIPERFECTO ${ }^{1}$
}

The mapudungun -fu morpheme: the grammatical coding of anti-perfect

\author{
Guillermo Soto* \\ Felipe Hasler**
}

Resumen

El perfecto es un aspecto de perspectiva retrospectiva que codifica la vigencia en el momento de habla de una eventualidad anterior. El presente trabajo propone la existencia de una categoría semántica y gramatical de antiperfecto que, en contraste con el perfecto, codifica la no vigencia en el momento de habla de una eventualidad pasada. Debido a que, al igual que el perfecto, el antiperfecto es sensible a la vigencia de una eventualidad anterior en el momento de habla, proponemos que se trata de un segundo tipo de aspecto de perspectiva retrospectiva. Tras revisar las nociones de perfecto en Comrie (1976), Dik (1997) y Nishiyama y Koenig (2010) y la noción de pasado discontinuo en Plungian y Van der Auwera (2006), se observa el modo en que la categoría propuesta se realiza, codificada gramaticalmente, en la lengua mapuche, mediante el morfema - $f u$, con especial énfasis en su caracterización semánticopragmática, su relación con la aktionsart y el papel del contexto en su interpretación.

Palabras clave: Antiperfecto, Mapudungun, Aspecto verbal, Contexto.

\section{Abstract}

The perfect aspect is a retrospective aspect that encodes the current relevance of a previous event. In this work, we propose a semantic and grammatical category labeled as antiperfect that, in contrast to the perfect aspect, encodes the non-validity of a previous eventuality in speech time. Given that, as the perfect aspect, the antiperfect is subject to the current relevance of a previous situation, we propose that this is a second type of retrospective aspect. After reviewing the descriptions of perfect by Comrie (1976), Dik (1997) and Nishiyama \& Koenig (2010), and the description of discontinuous past by Plungian \& van der Auwera (2006), we analyze how the antiperfect is grammatically encoded in Mapudungun by means of the morpheme $-f u$. We focus the analysis of this category on its semantic-pragmatic description, its relation to aktionsart, and the role of context in its interpretation.

Key words: Antiperfect, Mapudungun, Verbal aspect, Context.

\footnotetext{
${ }^{1}$ Los autores agradecen a Héctor Mariano por su invaluable colaboración en el presente estudio. Asimismo, agradecen a Lucía Golluscio y Gilberto Sánchez por sus comentarios en distintos momentos de la investigación que condujo a este trabajo. Los errores y deficiencias del trabajo son, por supuesto, de exclusiva responsabilidad de los autores. La investigación fue apoyada parcialmente por el proyecto Fondecyt 1110525.
} 


\section{INTRODUCCIÓN}

En las últimas décadas, estudios en diversas lenguas han venido reconociendo una noción semántica, codificada gramaticalmente, que, a nuestro entender, puede considerarse como un tipo de tiempo relativo o, mejor aún, de aspecto externo, no descrito satisfactoriamente todavía. Específicamente, se trata de una categoría gramatical expresada típicamente por un morfema $-\mathrm{o}$, alternativamente, por una construcción - que comunica que la eventualidad ${ }^{2}$ (en adelante, Ev) designada por la cláusula está desvinculada del momento de habla (Bertinetto, 1997; Hantson, 2005; Plungian y Van der Auwera, 2006; Salas, [1992] 2006; Soto y Hasler, 2010). Esta categoría no puede simplemente asimilarse a un tiempo pasado estándar, pues, como ya ha señalado Comrie (1985), en los tiempos pasados la no vinculación con el momento de habla se determina pragmáticamente y no forma parte del significado semántico de la unidad; más específicamente, puede caracterizarse como una implicatura pragmática guiada por la máxima de relevancia o pertinencia —en la línea de lo planteado por Golluscio (2000)_. Además, la categoría opera sobre predicados que ya tienen una referencia temporal asignada por otros mecanismos -ya gramaticales, ya pragmáticos - sin alterar, necesariamente, su referencia temporal. Un ejemplo de la categoría es el morfema $-f u$ del mapudungun ${ }^{3}$. En esta lengua se establece un contraste entre las cláusulas:

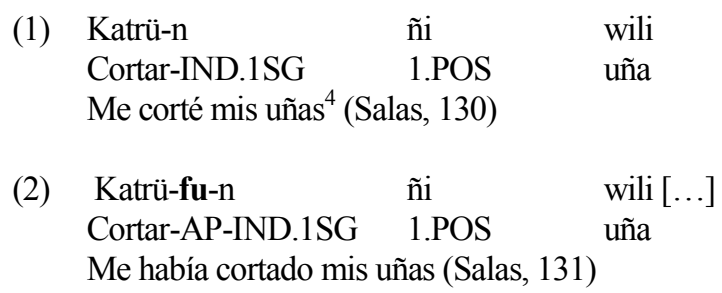

\footnotetext{
${ }^{2}$ Se entiende el término 'eventualidad' como sinónimo de lo que Dik (1997) llama 'estado de cosas' y Smith (1997) 'situación', es decir, "la concepción de algo que puede ser el caso en algún mundo".

${ }^{3}$ El mapudungun es una lengua genéticamente aislada, hablada con distintos grados de vitalidad en el centro y sur de Chile y Argentina. Se trata de una lengua aglutinante y sufijadora con tendencia a la polisíntesis. El orden no marcado de los elementos de una oración es SVO/VS. El verbo puede incluir un gran número de sufijos que expresan categorías como: tiempo, aspecto, modalidad, direccionalidad, evidencialidad, polaridad y, además, cuenta con procesos morfosintácticos como la incorporación nominal y reduplicación (para una descripción detallada de la estructura del mapudungun veáse Smeets [1989], 2008; Salas [1992], 2006; Zúñiga, 2006).

${ }^{4}$ Las abreviaturas utilizadas son: $1=1^{\mathrm{a}}$ persona, $2=2^{\mathrm{a}}$ persona, $3=3^{\mathrm{a}}$ persona, [ $]=$ forma no marcada, $\mathrm{ADM}=$ admirativo, $\mathrm{AP}=$ antiperfecto, $\mathrm{CONJ}=$ conjunción, $\mathrm{DEM} 1=$ demostrativo 1 (chi), DEM2= demostrativo $2(f e y)$, DET1 $=$ determinante $1(t a), \mathrm{DET} 2=$ determinante $2(t i), \mathrm{DIR}=$ direccional, DIST $=$ distributivo, $\mathrm{DU}=$ dual, $\mathrm{EST}=$ estativizador, $\mathrm{EVID}=$ evidencialidad, $\mathrm{FNF} 1=$ forma no finita $1(-l u), \mathrm{FNF} 2=$ forma no finita $2(-e l), \mathrm{FUT}=$ futuro, $\mathrm{HAB}=$ habitualidad, $\mathrm{IND}=$ indicativo, $\mathrm{NEG}=$ negación, $\mathrm{OP}=$ objeto primario, $\mathrm{PAS}=$ pasiva, $\mathrm{PPOS}=$ posposición, $\mathrm{POS}=$ posesivo, $\mathrm{PL}=$ plural, $\mathrm{SG}=$ singular, $\mathrm{POS}=$ posesivo.
} 
Mientras la primera se interpreta como un pasado, la segunda exige, adicionalmente, que el resultado de la Ev no se mantenga vigente en el momento de habla (p. ej., Me corté las uñas, pero me crecieron).

En un trabajo anterior (Soto y Hasler, 2010), hemos propuesto que la categoría recién presentada puede considerarse como un tipo de aspecto de perspectiva retrospectiva (Dik, 1997) cuyo significado es inverso al del perfecto, razón por la que la hemos denominado antiperfecto. Mientras el perfecto codifica la vigencia en el momento de habla de una Ev localizada temporalmente antes de dicho momento, el antiperfecto, de ser nuestra caracterización adecuada, codifica la no vigencia en el momento de habla de una Ev anterior. Como hemos mostrado en dicho trabajo, tres de los cuatro usos típicamente asociados al perfecto, a saber, resultativo, continuativo y pasado reciente ( $c f$. Comrie, 1976) tienen su inverso en el antiperfecto, que puede comunicar que cierto resultado esperable de una Ev dinámica no se dio (antirresultativo), que la persistencia en el momento de habla de una Ev estativa no es el caso (anticontinuativo) o que una Ev se localiza en un pasado remoto. Como en el caso del perfecto, parte de estos usos suponen cierta interacción entre la modalidad de acción verbal o aktionsart ${ }^{5}$ y el marcador de antiperfecto.

$\mathrm{Si}$ bien pensamos que el argumento a favor de la existencia de un antiperfecto como aspecto de perspectiva retrospectiva es sólido y tiene validez tipológica en tanto se aplica a lenguas no relacionadas, no hay hasta ahora caracterizaciones finas de sus propiedades semánticas y pragmáticas; en otras palabras, no parece haber una descripción de los factores semánticos y pragmáticos que entran en juego en su configuración. El presente trabajo tiene por objeto proponer una caracterización interna de la categoría. Luego de la introducción, en $\$ 2$ se define al antiperfecto como una categoría espejo del perfecto, que codifica la no vigencia de una Ev pasada en el momento de habla y se propone que la caracterización semántico-pragmática del perfecto de Nishiyama y Koenig (2010) puede extenderse, mutatis mutandi, al antiperfecto, en tanto en ambos casos se relaciona una Ev pasada con el momento de habla. Mientras con el perfecto el hablante vincula la Ev pasada con una Ev estativa vigente en el momento de habla; en el antiperfecto, el hablante cancela una eventual relación entre la Ev pasada y una Ev estativa vigente en el momento de habla que el oyente podría inferir. En $\S 3$ se propone que el morfema -fu corresponde a un marcador de antiperfecto sensible al tipo de Ev, específicamente, a las distinciones dinámico vs. estativo y, dentro de los estativos, estativos permanentes vs. contingentes. Por la presencia de un operador gramatical específico, el antiperfecto puede desencadenar cambios de tipo explícitos en la interpretación aspectual de la situación. Finalmente, en $\S 4$ se resumen las principales conclusiones de la presente investigación.

\footnotetext{
${ }^{5}$ El término aktionsart, asociado al aspecto léxico, se utiliza en este estudio para referirse al aspecto de la constelación verbal o predicación nuclear, siguiendo las propuestas de Dik (1997), "tipo de estado de cosas" y Smith (1997), "tipo de situación".
} 


\section{EL ASPECTO DE PERSPECTIVA RETROSPECTIVA}

\subsection{EL PERFECTO}

A medio camino entre la categoría gramatical de tiempo, que se ocupa de la localización de una Ev respecto de un momento de habla, y la de aspecto interno, que especifica las propiedades temporales internas de un Ev, se encuentra una categoría que codifica la relación que dos Evs establecen entre sí. Típicamente, esta categoría se manifiesta mediante nociones como la de perfecto (Juan se ha quebrado el brazo) o la de prospectivo (Los precios van a subir). Mientras unos autores han caracterizado la categoría como una forma de tiempo relativo (Comrie, 1976; Hengeveld y Mackenzie, 2008), también se la ha descrito como un tipo de aspecto externo (Dik, 1997). En todo caso, como puede desprenderse de Smith (1997), la categoría presenta propiedades tanto temporales como aspectuales. Más interesante aun es que con ella una Ev permite introducir otra, relacionada con la primera, en el discurso (Smith, 2003). Así, en la lectura de perfecto resultativo de Juan se ha quebrado un brazo, la Ev Juan quebrarse un brazo introduce una Ev parafraseable en Juan tiene el brazo quebrado, esto es, el estado resultante de quebrarse el brazo, que se mantiene vigente en el momento de habla. Ciertamente, el fenómeno es más complejo, toda vez que la misma cláusula puede tener una lectura de corte existencial en que el estado resultante no está vigente en el momento de habla: Juan se ha quebrado el brazo dos veces en su vida, pero ahora lo tiene sano. Por otro lado, también son posibles perfectos con Ev atélicas que pueden generar lecturas denominadas continuativas en que la misma Ev designada por el participio persiste en el momento de habla: He vivido toda mi vida en Chile. Además, el perfecto puede expresar la proximidad al momento de habla de un Ev, tanto en el llamado perfecto hodiernal: Hoy he visto a Andrea, como en el perfecto de noticia fresca (hot news): Ha muerto el rey (véanse Comrie, 1976 y Soto, 2012 para una caracterización de estas lecturas).

La existencia de diversas lecturas condicionadas, aparentemente, por factores semánticos como la aktionsart de la Ev, ha llevado a proponer la ausencia de un significado básico para el perfecto. Sin embargo, no es claro que las distintas interpretaciones del perfecto correspondan a tipos constreñidos semánticamente, como propone Comrie (1976). Más bien, siguiendo a Dahl (1985), puede pensarse que se trata de usos pragmáticamente condicionados. En efecto, una misma Ev admite más de una interpretación. Así, la cláusula He leído El Quijote puede usarse como resultativa, existencial o de pasado próximo: puede ser el caso que esté vigente en el momento de habla el conocimiento resultante de la lectura; que el hablante simplemente plantee que ha ocurrido la lectura al menos una vez en su vida (He leído el Quijote varias veces en mi vida) o que el hablante haya acabado de terminar de leer el libro. También una Ev atélica puede dar lugar, por ejemplo, a lecturas continuativas o existenciales. Así, He estado en este trabajo puede querer decir que el hablante aún está en el trabajo (He estado toda mi vida en este trabajo) o que el hablante, en algún momento de su vida, ha estado en el trabajo en cuestión (Ya he estado en este trabajo). 
Recientemente, Nishiyama y Koenig (2010) han propuesto una caracterización del perfecto que, junto con dar cuenta de la diversidad de usos, caracteriza un significado básico para la categoría. La idea de los autores es que el perfecto introduce dos Evs en el discurso, las que se relacionan epistémicamente entre sí: una Ev, descrita por el verbo principal y sus argumentos, y un estado (desde ahora, $\mathrm{S}$ ) que se superpone al momento de habla. S representa la vigencia de Ev en el momento de habla y es semánticamente inespecífico, en tanto su categoría es una variable libre cuyo valor específico debe ser determinado pragmáticamente. Debe precisarse que, para los autores, si bien el valor de la variable se fija de modo pragmático, su existencia forma parte de la semántica del perfecto.

De modo más específico, los autores sostienen que la determinación pragmática de $\mathrm{S}$ obedece a una inferencia generada a partir de la ocurrencia de Ev, siguiendo el principio de informatividad, o principio-I, que señala que lo que se expresa de manera simple se ejemplifica de modo estereotipado (Levinson, 2000). Este principio consta de una máxima de minimización, por parte del hablante, y de un enriquecimiento pragmático, por parte del oyente. En lo que respecta a la minimización, el hablante escoge el enunciado menos informativo - en este caso, el enunciado que porta la marca de perfecto - cuando hay uno más informativo disponible. Así, por ejemplo, el hablante expresa Marta se ha quebrado el brazo, en vez de Marta se quebró el brazo y lo tiene quebrado. El oyente, por su parte, enriquece el enunciado menos informativo con la interpretación más específica, utilizando su conocimiento de mundo y la información contextual, y aprovechando el contraste entre el perfecto y la forma canónica de pretérito indefinido para designar una Ev pasada. En otras palabras, debido a que existe una forma canónica, el pretérito indefinido $^{6}$, para introducir Evs pasadas, la opción del perfecto por el hablante desencadena la inferencia.

De lo anterior se desprende que la relación entre Ev y S es determinada por el hablante y no se puede interpretar independientemente de la evaluación que este hace de la situación. Esta parece ser la razón por la que Langacker (1987) señala que el perfecto es una construcción subjetiva en primer grado —o atenuada (Langacker, 2000) - : si bien la ocurrencia de Ev sigue perteneciendo al dominio objetivo de la situación, su vigencia presente, representada por S, no pertenece a la situación en sí misma, sino que es introducida por el conceptualizador (para una explicación más detallada de la relación entre las propuestas de Nishiyama y Koenig y Langacker, véase Soto, 2012).

Aunque la noción de subjetividad de Langacker permite caracterizar, desde una perspectiva lingüístico cognitiva, el proceso de conceptualización sobre el que descansa el perfecto, consideramos que, como ha destacado Verhagen (2005), no explicita suficientemente la dimensión interpersonal del proceso de conceptualización, que,

\footnotetext{
${ }^{6}$ Utilizamos el término pretérito indefinido y no pretérito perfecto simple para evitar la confusión con el significado perfecto.
} 
finalmente, resulta crítica para la coordinación de hablante y oyente en el intercambio comunicativo. En efecto, el conceptualizador debe considerar lo que el oyente puede ser capaz de reconstruir en la comunicación. De esta manera, Verhagen complementa la noción de subjetividad planteada por Langacker, señalando que cualquier evento lingüístico necesariamente involucra dos conceptualizadores, uno que tiene el rol de ser el responsable del enunciado, identificado por Langacker, y, además, otro, que lo interpreta de una forma particular: "el punto de un enunciado lingüístico, en términos generales, es que el primer conceptualizador invita al segundo a atender en conjunto un objeto de conceptualización en alguna manera específica y a actualizar la base común al hacerlo" (Verhagen, 7).

De acuerdo con lo anterior, podemos concluir que el hablante, al introducir el perfecto, introduce dos eventualidades, Ev y S, codificando solamente Ev, y le indica al oyente que debe buscar, en el contexto y en su conocimiento de mundo, un $\mathrm{S}$ que indique la vigencia de Ev en el momento de habla. Este proceso está mediado por el principio-I, de tal forma que el hablante realiza la minimización indicada por dicho principio, pues piensa que, por medio del contexto y de su conocimiento de mundo, el oyente será capaz de maximizar y reconocer la relación que construyó, es decir, que será capaz de encontrar un $\mathrm{S}$ que se relacione epistémicamente con Ev, que indique su vigencia en el momento de habla. Por tanto, pensamos que el perfecto no es una construcción subjetiva, sino más bien, intersubjetiva, donde los dos participantes en la interacción verbal, tanto el hablante como el oyente, juegan un papel primordial en la conceptualización de la situación.

\subsection{EL ANTIPERFECTO}

Como hemos propuesto en un trabajo anterior (Soto y Hasler, 2010), el antiperfecto es la categoría espejo del perfecto, no solo porque posee un significado inverso, sino también porque tres de los tipos de perfecto señalados por Comrie (1976), el continuativo, el resultativo y el reciente, tienen su correlato especular en el antiperfecto, registrándose casos de anticontinuativo (en español, mapudungun e inglés), antirresultativo y pasado remoto (en mapudungun). Además, resulta sugerente constatar que los tipos de antiperfecto señalados anteriormente se corresponden con las variantes de pasado discontinuo ${ }^{7}$ señaladas por Plungian y Van der Auwera (2006), denominadas 'pasado enmarcado', cuando se combina el marcador de pasado discontinuo con una situación imperfectiva, y 'pasado de resultado cancelado', cuando este se combina con una perfectiva. Consecuentemente, hemos planteado que el aspecto de perspectiva retrospectiva no se manifiesta solo en el perfecto, que codifica la vigencia de un EdC en el momento de habla, como propone Dik (1997), sino también

\footnotetext{
${ }^{7}$ A grandes rasgos, los autores señalan que los marcadores de pasado discontinuo son marcadores de tiempo pasado que portan un significado de 'pasado no presente' o 'pasado sin relevancia presente' (Plungian y Van der Auwera 317)
} 
en el antiperfecto, que codifica la no vigencia. Ambos tipos de aspecto de perspectiva son sensibles a la pertinencia de un EdC pasado en el momento de habla.

De esta manera, proponemos que el hablante, al utilizar un marcador de antiperfecto, también introduce dos eventualidades, Ev y S, las que tienen las mismas características que las eventualidades introducidas por el perfecto, con la diferencia de que la introducción de S cancela la vigencia de Ev en el momento de habla. Por lo tanto, consideramos que el antiperfecto es una construcción intersubjetiva, en la que el hablante realiza la minimización indicada en el principio-I, pues espera que el oyente maximice el enunciado, encontrando en el contexto un $\mathrm{S}$ que se relaciona epistémicamente con Ev y que cancela su vigencia en el momento de habla. De lo hasta aquí expuesto, se sigue que en el presente trabajo, a diferencia de Plungian y Van der Auwera, proponemos que los tipos de antiperfecto deben caracterizarse no solo semántica sino también pragmáticamente.

En la siguiente sección analizaremos el morfema - $f u$, perteneciente al sistema gramatical de la lengua mapuche, como un caso de antiperfecto codificado gramaticalmente, para así poder avanzar en la caracterización semántico-pragmática de la categoría propuesta.

\section{EL ANTIPERFECTO COMO CATEGORÍA GRAMATICAL: EL - FU DEL MAPUDUNGUN ${ }^{8}$}

\subsection{EL SISTEMA DE REFERENCIA TEMPORAL DEL MAPUDUNGUN}

Como ya hemos señalado, pensamos que el morfema - $f u$ codifica gramaticalmente el significado antiperfecto en mapudungun. Consecuentemente, no corresponde a un tipo de tiempo sino a un tipo de aspecto de perspectiva retrospectiva. Con el objeto de delimitar el significado del morfema $-f u$, analizaremos en primer lugar el sistema de referencia temporal de la lengua mapuche. Este se construye a partir de la dicotomía entre la forma no marcada y el morfema $-a$, que porta el significado de tiempo futuro ${ }^{9}$. En las cláusulas no marcadas con $-a$, la referencia temporal depende de

\footnotetext{
${ }^{8}$ A causa de que el antiperfecto es una categoría con un marcado componente subjetivo, es necesario precisar algunos aspectos de la variedad dialectal considerada en el estudio, ya que la subjetividad se suele asociar con una alta tendencia a la variación diatópica ( $c f$. Company, 2004). En el presente estudio se consideran mayoritariamente ejemplos pertenecientes al dialecto moluche. Según Salas [1992] (2006), la gran mayoría de los hablantes maneja esta variedad, la que se encuentra ubicada en la zona central y precordillerana de la IX Región. Salas ubica el dialecto picunche en el llano central de la provincia de Malleco, IX Región, y grupos aislados en la cordillera de Nahuelbuta, en la VIII Región. Por lo demás, el dialecto pehuenche se mantiene en la zona cordillerana comprendida entre el Alto Biobío, en la VIII Región, y la provincia de Valdivia, en la actual XIV Región. Finalmente, sitúa al dialecto huilliche en pequeñas agrupaciones ubicadas en la zona de Lago Ranco y San Juan de la Costa, X Región. Salas destaca, además, que más allá de las diferencias entre las variedades, aún se mantiene un alto nivel de inteligibilidad dialectal.

${ }^{9}$ No todos están de acuerdo en considerar a - $a$ como un morfema de futuro en mapudungun. Así, Hengeveld y Mackenzie (2008) proponen que se trataría de un morfema de modo. Nuestra propuesta es independiente del estatus temporal o modal de este morfema. Para el presente análisis no consideraremos los casos con morfema $-a$.
} 
la aktionsart de la predicación y el aspecto de punto de vista (Smith, 1997). Como se verá en lo que sigue, el determinante crítico de la referencia temporal es la aktionsart de la predicación, que puede ser dinámica o estativa.

Con predicados dinámicos, la Ev posee un aspecto de punto de vista perfectivo que comunica, por implicatura, una referencia pasada (Golluscio, 1998). La relación entre perfectividad y pasado, lejos de ser una singularidad del mapudungun, es un patrón general, fundado en la semejanza semántica de ambas nociones, que se manifiesta tanto sincrónica como diacrónicamente en distintas lenguas (Bybee et al, 1994). En el siguiente ejemplo, la $\mathrm{Ev}$ - correspondiente a una realización vendleriana, esto es, una situación dinámica, durativa y télica - sin marca temporal se interpreta como un pasado perfectivo:
(3) Dewma-n kiñe ruka
Hacer-IND.1SG un casa
Hice una casa $\left(\mathrm{HM}^{10}\right)$.

La misma interpretación pasada se obtiene con actividades y logros vendlerianos, lo que muestra que el rasgo pertinente es la dinamicidad: ${ }^{11}$

\begin{tabular}{|c|c|c|}
\hline María umawtu-y & ta $=\tilde{n} i$ & ruka mew \\
\hline María dormir-IND [3SG] & $\mathrm{DET} 1=1 . \mathrm{POS}$ & casa PPOS \\
\hline \multicolumn{3}{|c|}{ María durmió en mi casa (HM) } \\
\hline weda & ngürü & la-y \\
\hline malvado & zorro & Morir-IND [3SG] \\
\hline
\end{tabular}

Importa observar que la referencia temporal de las Ev dinámicas depende también del acto de habla, de modo que una Ev dinámica puede adquirir referencia temporal presente en actos de habla de fuerza ilocutiva directiva. Así, en órdenes o invitaciones:

$$
\begin{array}{ll}
\text { ¡Matetu-yu, } & \text { peñi! } \\
\text { Tomar mate-IND.2DU } & \text { hermano } \\
\text { Tomemos mate, hermano (HM). }
\end{array}
$$

En nuestro análisis nos concentraremos en los actos de habla declarativos. En lo que respecta a los predicados estativos, la situación es más compleja y depende del carácter permanente o episódico del estado (Smith, 1997; RAE, 2009). Con predicados estativos permanentes, la cláusula no marcada presenta un aspecto de punto de vista imperfectivo que comunica, típicamente, que el estado persiste en el

\footnotetext{
10 Los ejemplos marcados con HM fueron proporcionados por Héctor Mariano, hablante nativo de mapudungun y profesor de dicha lengua, en sesiones de elicitación.

${ }^{11}$ Las actividades son eventos dinámicos durativos y atélicos; los logros son dinámicos, no durativos y télicos (Vendler, 1967 y Smith, 1997).
} 
momento de habla, adquiriendo, vía implicatura, una referencia presente ( $c f$. Bybee et al, 1994), como se puede observar en el siguiente ejemplo:

$$
\begin{aligned}
& \text { Ta=ñi ruka müle-y willi mapu mew } \\
& \text { DET1=1.POS casa estar-IND.[3SG] sur tierra } \\
& \text { Mi casa está en el sur (HM). }
\end{aligned}
$$

Por su parte, los predicados estativos episódicos se presentan como perfectivos y generan, por tanto, una implicatura de referencia temporal pasada ${ }^{12}$.

(8) Rupachi semana mew kütran-küle-n feymew küdaw-me-la-n

Pasada semana PPOS enfermo-EST-IND.1SG entonces trabajar-

\section{DIR-NEG-IND.1SG}

La semana pasada estaba enfermo así que no fui a trabajar (HM).

(9) ¿Chew müle-ymi sábado antü mew?

Dónde estar-IND.2SG sábado día PPOS

¿Dónde estabas el día sábado? (HM)

También, el sistema gramatical mapuche permite que predicados episódicos y eventos se recategoricen como estativos permanentes derivados, modificando, por tanto, su referencia temporal, gracias al empleo del morfema de habitualidad -ke, desencadenante, en este caso, de un cambio de tipo explícito:
(10) $\mathrm{Ka}$ mawida mew pe-ke-y engün dañinentu
CONJ monte PPOS ver-HAB-IND.[3] PL mutilla
ka fill anümka
CONJ todoárbol

También en la montaña observan la mutilla y toda clase de árboles (Aguilera et al, 1).

En síntesis, el mapudungun, al menos en lo que respecta al pasado y el presente, no posee morfemas de tiempo. La referencia temporal se establece pragmáticamente a partir del modo de acción de la Ev y el aspecto de punto de vista asociado por defecto a cada modo de acción. Como se observa en el caso del morfema -ke, la variación en la aktionsart conlleva el cambio de la referencia temporal.

\footnotetext{
${ }^{12}$ Es posible que la interpretación perfectiva del predicado estativo episódico obedezca a que este se conceptualiza como dinámico, esto es, como un evento, a diferencia del permanente. De ser esta interpretación correcta, en mapudungun, los estativos episódicos se interpretarían típicamente como eventos.
} 


\subsection{EL MORFEMA -FU Y LA RELACIÓN DE LOS PREDICADOS CON EL MOMENTO DE HABLA}

La inserción del morfema $-f u$ en predicaciones que, como hemos visto, ya poseen referencia temporal, altera cierta relación que los predicados tienen con el momento de habla. Esta relación, como veremos, no siempre es temporal, y se vincula con la modalidad de acción de Ev.

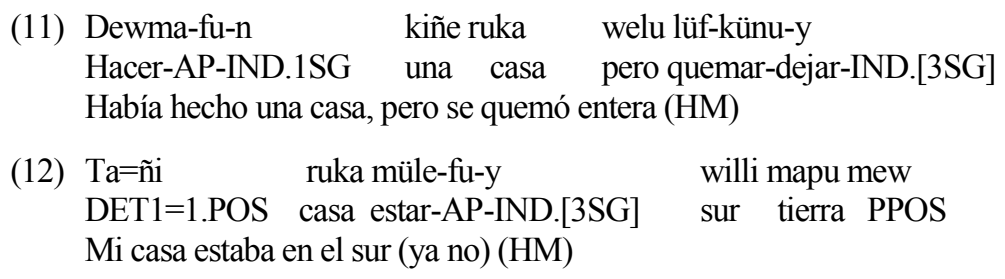

El ejemplo (11) que contiene la Ev dinámica 'hacer una casa' contrasta con el ejemplo (3), que contiene la misma Ev. La diferencia entre ambas cláusulas no es temporal, toda vez que las dos refieren a una eventualidad pasada: en los dos casos se hizo una casa en un tiempo anterior al momento de habla. La diferencia radica en que en (11) - $f u$ comunica que no se sostiene en el momento de habla un estado resultante esperable de la Ev dinámica. Por su parte (12), que contiene una Ev estativa permanente, contrasta temporalmente con (7), que contiene la misma Ev, en tanto su referencia no es presente, sino pasada. De acuerdo con nuestro análisis, el cambio de referencia temporal obedece a que en (12) -fu codifica la cancelación de la implicatura conversacional generalizada que permite la referencia temporal presente a partir de un estativo permanente. Abstrayendo las diferencias derivadas de la aktionsart de (11) y (12), se puede plantear que en ambos casos -fu marca la no vigencia en el momento de habla de un estado esperable a partir de la Ev designada por la cláusula. Esta interpretación es equivalente a la noción de implicatura rota de Golluscio (2000).

Como hemos visto, el morfema $-f u$ desvincula la Ev anterior del momento de habla, codificando su no vigencia. En un nivel de especificidad mayor pueden distinguirse dos tipos de -fu correlativos a los tipos de perfecto de Comrie (1976). En primer lugar, un anticontinuativo que designa Evs de duración limitada, que no se extienden hasta el momento de habla, casos como (12). Segundo, un antirresultativo que denota la no existencia de un estado consecuente en el momento de habla (o la no relevancia de dicho estado en el momento de habla), casos como (11).

La correlación entre la aktionsart de Ev y el tipo de antiperfecto es estrecha. En un análisis de 108 cláusulas con morfema $-f u^{13}$ hemos encontrado que en los casos

\footnotetext{
${ }^{13}$ Las cláusulas fueron tomadas de dos fuentes. En primer lugar, de los epews: Sumpall, Trülke wekufü, Mangkian, Un viaje al país de los difuntos, Un viaje de los difuntos al país de los vivientes, La mujer bruja y su hijo y El zorro y el huillín, todos presentes en Salas [1992] (2006). En segundo lugar, de los nütram 
con estados permanentes predomina el antiperfecto anticontinuativo, mientras que en los casos con Ev estativa episódica y Ev dinámica predomina el antiperfecto antirresultativo. El siguiente cuadro sintetiza los datos:

\begin{tabular}{|l|c|c|}
\hline Modalidad de acción de Ev & Anticontinuativo & Antirresultativo \\
\hline Estativo permanente & 77 & 4 \\
\hline Estativo episódico & 0 & 2 \\
\hline Dinámico & 0 & 25 \\
\hline
\end{tabular}

Cuadro 1: Correlación entre aktionsart de la Ev y tipo de antiperfecto

Puesto que el antiperfecto anticontinuativo se vincula con Ev estativas permanentes, esta resulta ser su interpretación no marcada, mientras que la intepretación no marcada de un antiperfecto con Ev dinámica o estativa episódica es la de antirresultativo. En la sección siguiente analizaremos tanto dichas interpretaciones como las condiciones que posibilitan el surgimiento de las interpretaciones marcadas.

\subsubsection{El ANTICONTINUATIVO}

En virtud de que el anticontinuativo resulta de la interacción entre el morfema $f u$ y una Ev estativa permanente, la construcción típicamente anticontinuativa se da con verbos con aktionsart básica estativa permanente:

(13) Kiñe rupachi kiñe domo kalko-rke nie-rke-fu-y Una vez una mujer bruja-ADM tener-EVID-AP-IND.[3SG] alün püñeñ. mucho hijo (de la mujer)

Había una vez una mujer, que resultó ser bruja, que tenía muchos hijos (Salas, 257).

No obstante lo anterior, también es posible encontrar cláusulas que, presentando verbos con aktionsart básica dinámica, poseen significado anticontinuativo, como en:

$$
\begin{array}{llll}
\text { Faw püle } & \text { rupa-ke-fu-y } & \text { ko piam } & \text { kuyfi } \\
\text { Acá PPOS } & \text { pasar-HAB-AP-IND.3SG } & \text { agua EVID } & \text { antes } \\
\text { Por acá pasaba un río antes (pero ya no). (HM) } & &
\end{array}
$$

Fey ti wentru müna putufe nge-fu-y

DEM2 DET2 hombre muy bebedor ser-AP-IND.[3SG]

Labranza amu-ke-fu-y puliwentu

Labranza ir-HAB-AP-IND.3SG mañana

2, 4, 5, 6, 7, 8, 10, 11, 12, 15, 16, 17 y 18 presentes en Relmuan (1997). Para el análisis consideramos solo las apariciones de -fu en cláusulas independientes en modo indicativo con el objeto de observar de mejor manera su relación con la aktionsart. Una proyección de la presente investigación es observar su relación tanto con el marcador de tiemp o modo - $a$ y con el marcador de subjuntivo - $l$. 
Ese hombre era muy bebedor; iba todas las mañanas a Labranza (Relmuan, 21).

Lo anterior es posible porque los verbos dinámicos (rupa- 'pasar' y amu- 'ir') van acompañados por el morfema - $k e$, que funciona como un marcador de habitualidad. El morfema -ke produce un cambio explícito en la aktionsart de las constelaciones verbales dinámicas, transformándolas en Evs estativas habituales derivadas que, en combinación con el morfema - $f u$, adquieren un significado anticontinuativo. El morfema $-k e$, al estativizar las constelaciones verbales dinámicas, les agrega la propiedad de subintervalo (Smith, 1997), generando, consecuentemente, la implicatura de que la situación continúa hasta el presente. Esta implicatura es cancelada por el morfema - $f u$. Si nuestra interpretación es correcta, las cláusulas con verbos dinámicos y morfema -ke deberían tener referencia temporal presente, como las cláusulas con verbos estativos permanentes, lo que, como se observa en el ejemplo (10), efectivamente ocurre.

El análisis expuesto explica por qué se genera la interpretación anticontinuativa no solo con verbos estativos permanentes, sino también con dinámicos, como se observa en el Cuadro 2, que sintetiza la relación entre el tipo de situación básica del verbo y el antiperfecto anticontinuativo: los 40 verbos dinámicos que aparecen en cláusulas con interpretación anticontinuativa están en combinación con el morfema $-k e$, que funciona como un estativizador de constelaciones verbales dinámicas.

\begin{tabular}{|l|l|l|}
\hline & Estativos & Dinámicos \\
\hline Anticontinuativo & 37 & 40 \\
\hline
\end{tabular}

Cuadro 2: Relación entre antiperfecto anticontinuativo y tipo de situación básica del verbo.

En síntesis, en la interpretación anticontinuativa el hablante introduce una Ev estativa, básica o derivada, marcada con el morfema - $f u$, y espera que el oyente encuentre un $\mathrm{S}$, en el contexto y en su conocimiento de mundo, que indique que $\mathrm{Ev}$ no persiste hasta el momento de habla.

\subsubsection{EL ANTIRRESULTATIVO}

El antirresultativo obedece, típicamente, a la combinación del morfema - $f u$ con constelaciones verbales dinámicas. En este tipo, como ya hemos dicho, el morfema no altera la relación temporal que el predicado establece con el momento de habla, sino que anula una relación posible entre la Ev pasada y el momento de habla, como se puede ver en el ejemplo (2), repetido a continuación como (16):

(16) Katrü-fu-n ñi wili

Cortar-AP-IND.1SG 1.POS uña

Me había cortado las uñas (pero me volvieron a crecer) (Salas 131). 
El antirresultativo no solo comunica que un resultado estricto no se da en el momento de habla, sino que puede también indicar que la acción realizada no tuvo las consecuencias esperadas:
(17) Kintu-fu-y engün fey-chi epu pichi-ke domo Buscar-AP-IND [3] PL DEM2-DEM1 dos pequeño-DIST mujer welu pe-la-fi-y engün. pero ver-NEG-3.OP-IND.[3] PL Habían buscado a las 2 niñas, pero no las encontraron (Salas 249).

Además, puede comunicar que una consecuencia esperable de un determinado estado episódico no se verificó, como por ejemplo:
(18) Juan kim-nie-fu-y ta=ñi mawü-a-el, Juan saber-tener-AP-IND.[3SG] DET1=3.POS llover-FUT-FNF2 welu tripa-y müten pero salir-IND.[3SG] no más
Juan sabía que iba a llover, pero salió no más (HM).
(19) Rupachi semana mew Pasada semana PPOS kutran-küle-fu-n welu küdaw-me-n müten trabajar-DIR-IND.1SG no más La semana pasada estuve enfermo pero fui a trabajar igual (HM).

Finalmente, es frecuente encontrar el morfema - $f u$ en combinación con verbos de comunicación, indicando que ciertos efectos esperados de un acto comunicativo no se dieron y, consecuentemente, no están vigentes en el momento de habla:
(20) Ngülam-nge-fu-y, allkü-la-y engün dew aconsejar-PAS-AP-IND.[3SG] escuchar-NEG-IND.[3] PL ya fütra trem-lu engün ka grande crecer-FNF1 PL CONJ

Se les ha aconsejado, pero no han escuchado, como ya han crecido (Relmuan, 53-54).

En síntesis, la interpretación antirresultativa puede ser tanto estricta - el resultado léxicamente implicado por una Ev télica - como amplia — un estado posterior epistémicamente vinculado a la Ev e inferible en el contexto-. El paralelo con el perfecto resultativo, como este se describe en Comrie (1976) o Nishiyama y Koenig (2010), es claro.

Respecto del contexto, resulta interesante destacar que los datos sugieren que la aparición de $-f u$ en una cláusula se correlaciona estrechamente con la presencia de una cláusula coordinada adversativa, ya sea explícita (17-20) o recuperable del contexto (16), que comunica el S específico que cancela la vigencia de la Ev en el momento de habla. 


\subsubsection{ESTATIVOS PERMANENTES ANTIRRESULTATIVOS}

Finalmente, al menos en la variedad dialectal considerada en la presente investigación, la lectura antirresultativa también puede surgir a partir de Evs estativas permanentes, aunque en un contexto específico. Si bien, como hemos indicado con anterioridad, al combinarse con $-f u$, las constelaciones verbales estativas permanentes reciben una interpretación no marcada de antiperfecto anticontinuativo, en ciertos contextos las cláusulas pueden tener interpretación antirresultativa amplia. En los siguientes ejemplos, una misma cláusula con Ev estativa permanente recibe interpretación anticontinuativa o antirresultativa amplia, dependiendo del contexto.

(21) Ni malle nie-fu-y kiñe mansun müten

1.POS tío tener-AP-IND.[3SG] un buey no más

Mi tío tenía un solo buey (HM).

(22) $\tilde{\mathrm{N}} \mathrm{i}$ malle nie-fu-y kiñe mansun welu

1.POS tío tener-AP-IND.[3SG] un buey pero

pepi-ngilla-la-y kangelu ñi

poder comprar-NEG-IND.[3SG] otro 1.POS

nie-a-el kiñe trarin

tener-FUT-FNF2 una yunta

Mi tío tiene un buey, pero no puede comprar otro para tener una yunta (HM).

Mientras la interpretación anticontinuativa presente en (21) surge sin mayores especificaciones contextuales, la interpretación antirresultativa amplia de (22) solo se da en el contexto de una oración adversativa en que la segunda cláusula explicita el estado no vigente en el momento de habla. Aun cuando nuestros datos muestran que las cláusulas antirresultativas con Ev dinámicas y con Ev estativas episódicas también se dan solo en oraciones adversativas, el punto es que, en el caso de las estativas permanentes, la ausencia de contexto adversativo impone la interpretación anticontinuativa. De hecho, no encontramos cláusulas con significado antiperfecto anticontinuativo en contextos adversativos. El antiperfecto antirresultativo con Ev estativas permanentes se da, aunque esporádicamente, en los datos analizados para el presente trabajo:

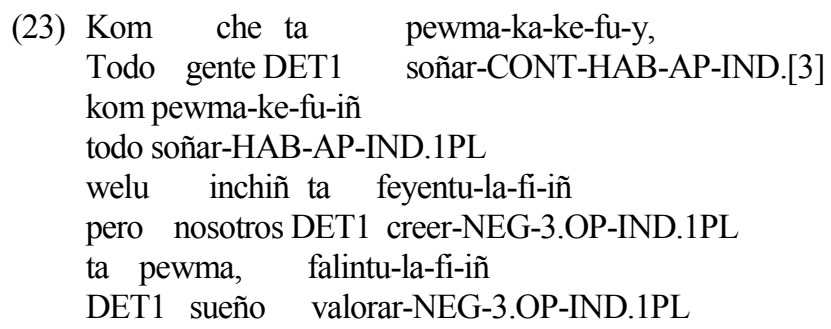


Todas las personas sueñan, todos nosotros soñamos siempre, pero nosotros no hemos creído en ellos, no les damos valor (Relmuan, 49).

En este último ejemplo el hablante emplea el verbo pewman 'soñar', marcado por el morfema de habitualidad y el de antiperfecto. En contexto adversativo, recibe una interpretación antirresultativa amplia: el hablante introduce la Ev estativa permanente para que el oyente introduzca el estado 'ya no son interpretados, no son valorados', que niega la vigencia de la primera Ev en el momento de habla. Un aspecto interesante de estos casos es que en ellos se conserva la referencia temporal presente propia de las cláusulas con Ev estativa permanente, lo que indica que no se coerciona la aktionsart de la predicación.

\subsubsection{EL PASADO REMOTO}

Además, como hemos señalado anteriormente, el morfema -fu también puede adquirir el valor de pasado remoto, en ocasiones complementado por adverbio el kuyfi 'hace mucho tiempo' o el morfema -rke, marcador de evidencialidad. Se presenta, fundamentalmente, en narraciones y en conversaciones que tratan acerca de las costumbres antiguas.

(24) Kuyfi antiguo mew, tripa-ke-rke-fu-y Antes antiguo PPOS salir-HAB-EVID-AP-IND.[3SG] la-püllel mew petu moro-le-lu mapu. cementerio PPOS todavía moro-EST-FNF1 tierra Antiguamente, en tiempos antiguos, se cuenta que salían los muertos del cementerio, cuando todavía era mora la tierra (o sea, antes de la llegada de los extranjeros cristianos) (Salas 253).

En estos casos, el morfema antiperfecto opera sobre una Ev estativa básica o, como en el ejemplo, derivada.

\section{CONCLUSIONES}

En el presente trabajo hemos propuesto una caracterización relativamente detallada del funcionamiento del morfema - $f u$ del mapudungun en su variedad moluche (Chile). De acuerdo con nuestro análisis, este morfema codifica un significado inverso al del perfecto, que denominamos antiperfecto. Mientras el perfecto codifica la vinculación con el momento de habla de una Ev iniciada con anterioridad a este, el antiperfecto codifica la desvinculación de una Ev iniciada con anterioridad al momento de habla. En nuestro análisis, proyectamos al antiperfecto la caracterización semántico-pragmática del perfecto propuesta por Nishiyama y Koenig (2010). De acuerdo con este análisis, el morfema - fu introduce dos Evs vinculadas epistémicamente entre sí, cuya relación está modelada por el principio-I. La diferencia con el perfecto es que en el antiperfecto la inferencia del oyente a partir de la Ev marcada con $-f u$ consiste en la no vigencia en el momento de habla de un estado 
esperable. Los usos del perfecto, salvo el experiencial, tienen su contraparte en los usos antiperfectos antes expuestos: anticontinuativo, antirresultativo y remoto.

El tipo específico de antiperfecto resulta de la interacción entre la modalidad de acción o aktionsart de la Ev marcada con -fu, el contexto y el empleo del marcador de antiperfecto. Con Ev dinámicas y estativas episódicas, -fu mantiene la referencia temporal pasada de la cláusula y genera una interpretación antiresultativa. Solo con Ev dinámicas télicas es posible que se den antirresultativas estrictas. Por su parte, con Ev estativas permanentes, sean estas básicas o derivadas, $-f u$ prototípicamente genera una interpretación anticontinuativa $\mathrm{y}$, en consecuencia, modifica la referencia temporal de la cláusula, que de presente cambia a pasado.

De modo esporádico, $-f u$ puede generar interpretación antirresultativa con estativos permanentes. Se trata de una interpretación marcada que exige un contexto adversativo en que la cláusula sin el marcador comunique — directa o indirectamenteel estado cancelado. En este caso, el morfema no modifica la referencia temporal de la cláusula que, al ser estativa permanente, sigue siendo presente.

La caracterización expuesta permite generalizar ciertas propiedades del antiperfecto antirresultativo y el anticontinuativo. El antiperfecto antirresultativo no modifica la referencia temporal de la cláusula, tal que, si esta es pasada, sigue siendo pasada, y si es presente - como ocurre en los casos con Ev estativa permanentesigue siendo presente. Junto con ello, el antiperfecto antirresultativo exige un contexto adversativo. Por su parte, el antiperfecto anticontinuativo modifica la referencia temporal de la cláusula, de presente a pasado. Es posible que de esto se deriven el empleo del antiperfecto como pasado remoto y, consecuentemente, la categorización que diversos autores han realizado del morfema como marca de tiempo gramatical. También parece ser esta la razón de que hablantes de español que aprenden mapudungun como segunda lengua, tiendan a emplear $-f u$ como morfema de tiempo pasado.

Universidad de Chile* Ignacio Carrera Pinto 1025, Ñuñoa, Santiago de Chile gsoto@uchile.cl

Universidad de Chile** CONICET (Consejo Nacional de Investigaciones Cientificas y Técnicas, Argentina) Ignacio Carrera Pinto 1025, Nuñoa, Santiago de Chile keche.felipe@gmail.com 
El morfema -fu del mapudungun: la codificación gramatical del antiperfecto

\section{OBRAS CITADAS}

Aguilera, Pedro; Ancán, José; Blanco, José y Víctor Pranao. Pu Mapuche Tañi Kimün. Temuco: Kume Dungu, 1987.

Bertinetto, Pier Marco. Il dominio tempo-aspettuale. Turín: Rosenberg \& Sellier, 1997. Bybee, Joan L., Revere, Perkins y William Pagliuca. The evolution of grammar: Tense, aspect and modality in the languages of the world. Chicago: The University of Chicago Press, 1994.

Company, Concepción. ¿Gramaticalización o desgramaticalización? El reanálisis y subjetivización de verbos como marcadores discursivos en la historia del español. Revista de Filología Española 2004: 29-66.

Comrie, Bernard. Tense. Cambridge: Cambridge University Press, 1985. Aspect. An introduction to the study of verbal aspect and related problems. Cambridge: Cambridge University Press, 1976.

Dahl, Östen. Tense and aspect systems. Nueva York: Basil Blackwell, 1985.

Dik, Simon. The Theory of Functional Grammar, Parte 1: The Structure of the Clause. Ed. Kees Hengeveld. Berlín y Nueva York: Mouton de Gruyter, 1997.

Golluscio, Lucía. "Rupturing implicature in the Mapudungun verbal system: the suffix -Fi". Journal of Pragmatics, 2000: 239-263.

_ "Aspecto Verbal en Mapudungun". Lingüistica y Literatura Mapuche. Aproximaciones desde ambos lados de los Andes. Ed. Lucía Golluscio y Yosuke Kuramochi. Temuco-Buenos Aires: Universidad Católica de Temuco, Universidad de Buenos Aires, 1998: 34-47.

Hantson, André. The English perfect and the anti-perfect used to viewed from a comparative perspective. Revista English Studies, 2005:245-268.

Hengeveld, Kees y Lachlan Mackenzie. Functional Discourse Grammar. A typologically-based theory of language structure. Oxford: Oxford University Press, 2008.

Langacker, Ronald. Grammar and Conceptualization. Berlín: Mouton de Gruyter, 2000.

— Foundations of cognitive grammar: theoretical prerequisites. Stanford, CA: Stanford University Press, 1987.

Nishiyama, Atsuko y Jean-Pierre Koenig (2010). What is a perfect state? Language. 2010: 611-646.

Levinson, Stephen. Presumptive Meanings: The Theory of Generalized Conversational Implicature. Cambridge: MIT Press, 2000.

Plungian, Vladimir y Johan van der Auwera (2006). "Towards a tipology of discontinuos past marking". Sprachtypologie und Universalienforschung. 2006:317-349.

RAE (Real Academia Española). Nueva gramática de la lengua española. Madrid: Espasa, 2009. 
Relmuan, María Angélica. Kiñeke nütram ka pentukun dungu feypiel pu Rapawe ka Rukapangui lof che. Temuco: UFRO, Instituto de Estudios Indígenas, 1997.

Salas, Adalberto. El mapuche mapuche o araucano. Fonología, gramática y antología de cuentos. Ed. Fernando Zúñiga. Santiago: Centro de Estudios Públicos, [1992] 2006.

Smeets, Ineke. A grammar of mapuche. Berlín: Mouton de Gruyter [1989] 2008.

Smith, Carlota. Modes of discourse. Cambridge: Cambridge University Press, 2003.

— The parameter of aspect. Dordrecht: Kluwer. Segunda edición [1991] 1997.

Soto, Guillermo. Los tiempos compuestos en el español de América. Tiempo, aspecto y uso. Tesis doctoral. Valladolid: Universidad de Valladolid, 2012.

Soto, Guillermo y Felipe Hasler. "La no vigencia en el momento de habla. Sobre la posibilidad del antiperfecto como categoría gramatical". La renovación de la palabra en el bicentenario de la Argentina. Los colores de la mirada lingüística. Ed. V. Castel y L. Cubo de Severino. Mendoza: Editorial FFyL, UN Cuyo, 2010: 1265-1271.

Vendler, Zeno. Verbs and times. Linguistics in philosophy. 1967:97-121. Nueva York: Cornell University Press.

Verhagen, Arie. Constructions of intersubjectivity. Oxford: Oxford University Press, 2005.

Zúñiga, Fernando. Mapudungun. El habla mapuche. Santiago: Centro de Estudios Públicos, 2006. 